Supporting Information for

\title{
Flexible and Patterned Thin Film Polarizer: Photo- polymerization of Perylene-based Lyotropic Chromonic Reactive Mesogens
}

Pureun Im, ${ }^{\dagger}$ Dong-Gue Kang, ${ }^{\dagger}$ Dae-Yoon Kim, ${ }^{\dagger}$ Yu-Jin Choi, ${ }^{\dagger}$ Won-Jin Yoon, ${ }^{\dagger}$ MyongHoon Lee, ${ }^{\dagger}$ In-Hwan Lee, ${ }^{\dagger}$ Cheul-Ro Lee, ${ }^{\dagger}$ and Kwang-Un Jeong ${ }^{*},{ }^{\dagger}$

${ }^{\dagger}$ Polymer Materials Fusion Research Center \& Department of Polymer-Nano Science and Technology, Chonbuk National University, Jeonju, Jeonbuk 561-756, Korea.

* Division of Advanced Materials Engineering, Chonbuk National University, Jeonju, Jeonbuk 561-756, Korea

* To whom the correspondence should be addressed. E-mail: kujeong@jbnu.ac.kr 
All reactants and reagent materials for synthesis were purchased from Sigma Aldrich and TCI. These materials were used as received without further purifications. The solvents such as DMF and THF were purified and water was deionized before use. Perylene derivatives (PDI-1, 2 and DAPDI) were synthesized according to the literatures. Chemical structure and purity of the synthesized molecule were identified by ${ }^{1} \mathrm{H}$ NMR in deuterated water $\left(\mathrm{D}_{2} \mathrm{O}\right)$ at $70{ }^{\circ} \mathrm{C}$ (Figure S1). Even though the DAPDI aqueous solution was heated, the stacking and aggregation still exist which leads to broad signals. To determine the molecular weight of the DAPDI, ESI-MS was used (Figure S2). The molecular weight of the molecule is $758.6 \mathrm{~g} / \mathrm{mol}$ and this value was well matched with its calculated value of $758.3 \mathrm{~g} / \mathrm{mol}$. Note that the bromide ion combining the amine group was not detected.

To obtain all available diffractions for unpolymerized crystals, the 1D WAXD pattern was measured for the DAPDI powder. From the 1D WAXD pattern (Figure S3), the $2 \theta$ value was converted to d-spacing. Chemical stability of the film was estimated by conducting the solvent resistance test which was dipping film into the various solvents for $5 \mathrm{~min}$. Samples were photo-polymerized via the identical process described in the Manuscript and the size of films were prepared to be $1 \mathrm{~cm}^{2}$. The photographic images after the solvent resistance test were shown a remarkable difference between bare and MAPT-modified substrates and the corresponding images were represented in Figure S4. The solvent resistance of film on the MAPT-modified substrate was improved compared with those of bare substrates. Note that all the photo-polymerized DAPDI TFPs were more stable than the non-polymerized pristine films. To fabricate the patterned TFP, the DAPDI- $\mathrm{H}_{2} \mathrm{O}(25: 75)$ solution was coated on the substrates by the simple doctor blade coating methods and dried at $25{ }^{\circ} \mathrm{C}$ for $24 \mathrm{~h}$. The coated films were covered with a photo-mask and irradiated with the UV light $\left(20 \mathrm{~mW} / \mathrm{cm}^{2}\right)$ for 30 min. The photo-polymerized DAPDI TFP was developed in methanol for 10-80 s. To eliminate defects generated on the bare glass and PET substrates, the two-step surface 
modification proceeded to the substrates: the generation of hydroxy functions by oxygen plasma and the grafting reaction of the photo-reactive molecule on the surface of the substrate (methacryloxypropyl-trimethoxysilane, MAPT). The detail schematic procedure and the structure of the reactive molecules were represented in Figure S5. The glass and PET substrates were washed with water, acetone and isopropyl alcohol before drying in the vacuum oven for $5 \mathrm{~h}$. For the oxidation of the surface, the oxygen plasma was conducted to the substrates with plasma cleaner (PDC-32G, Harrick plasma) for 5 min. The plasma treated surface was immediately dipped into the ethanol solution with $10 \mathrm{wt} \%$ MAPT for $1 \mathrm{~h}$. The MAPT-modified glass and PET substrates were washed with ethanol for several times and dried in the vacuum oven for $12 \mathrm{~h}$.

The morphology of positively patterned DAPDI TFPs on the bare and MAPTmodified glass substrate were observed using optical microscopic techniques and the corresponding images were shown Figure S6. The dotted- and striped-patterns were successfully constructed and polarizability was maintained even after the lithographic process. After the surface modification, the pattern was well formed without delamination. To investigate the surface topology of the patterned DAPDI TFP, AFM technique was conducted. The MAPT-modified surface was studied at the smaller scale (Figure 7d). As shown Figure S7, many small protrusions and thick grooves were observed along the SD. 


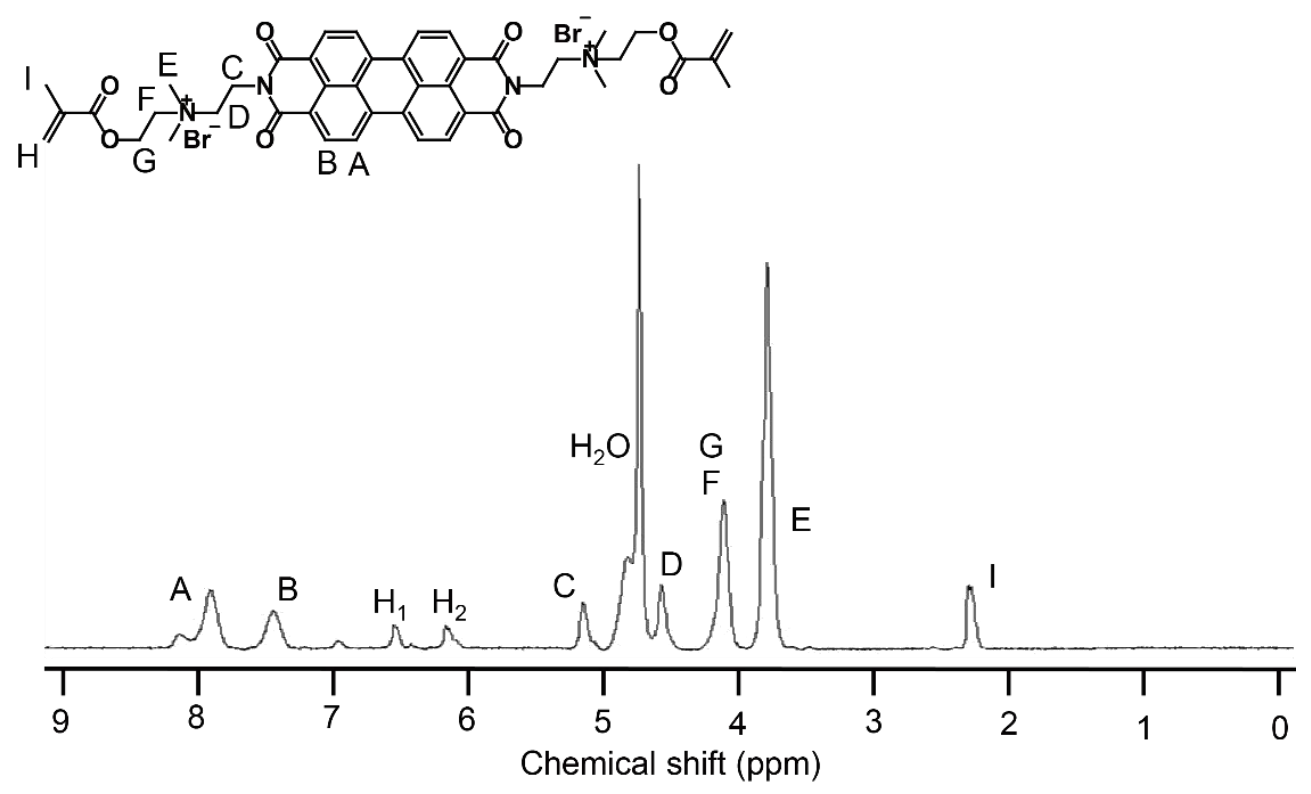

Figure S1. ${ }^{1} \mathrm{H}$ NMR spectra of DAPDI. 


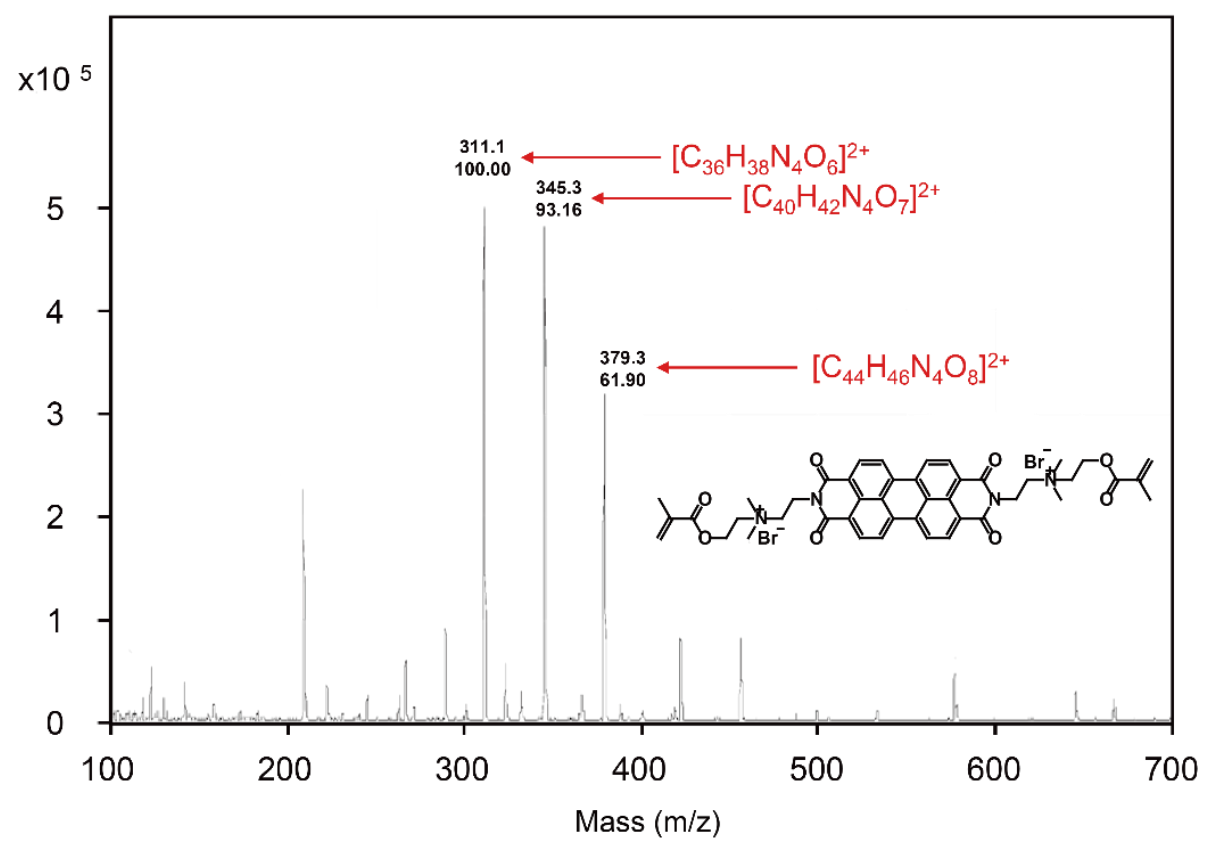

Figure S2. ESI MS of spectra of DAPDI. 


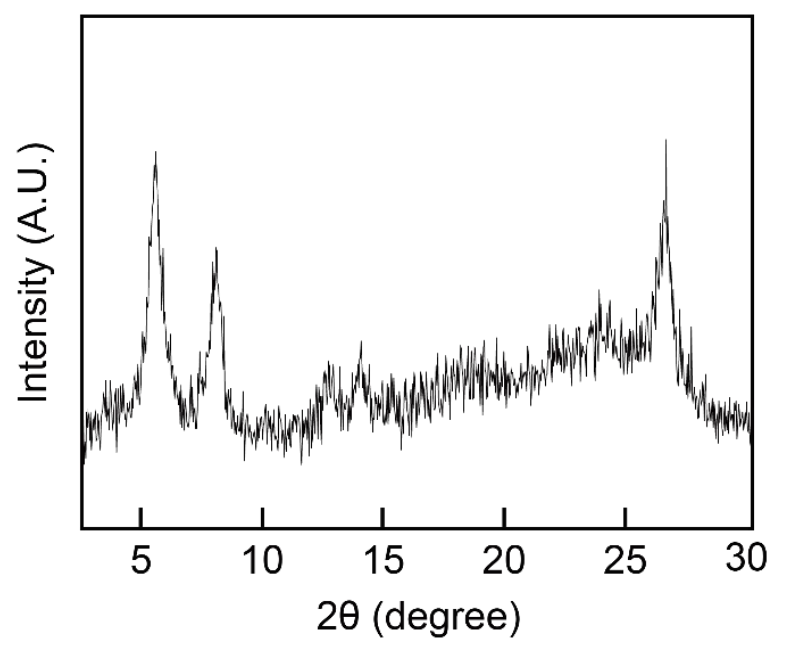

\begin{tabular}{cc}
\hline $\begin{array}{c}2 \theta \\
\text { (degree) }\end{array}$ & $\begin{array}{c}\text { d-spacing } \\
(\mathrm{nm})\end{array}$ \\
\hline 5.46 & 1.62 \\
8.05 & 1.09 \\
12.56 & 0.7 \\
13.9 & 0.63 \\
18.33 & 0.48 \\
23.73 & 0.37 \\
26.55 & 0.34 \\
\hline
\end{tabular}

Figure S3. 1D WAXD of DAPDI powder and its corresponding crystallographic parameters. 


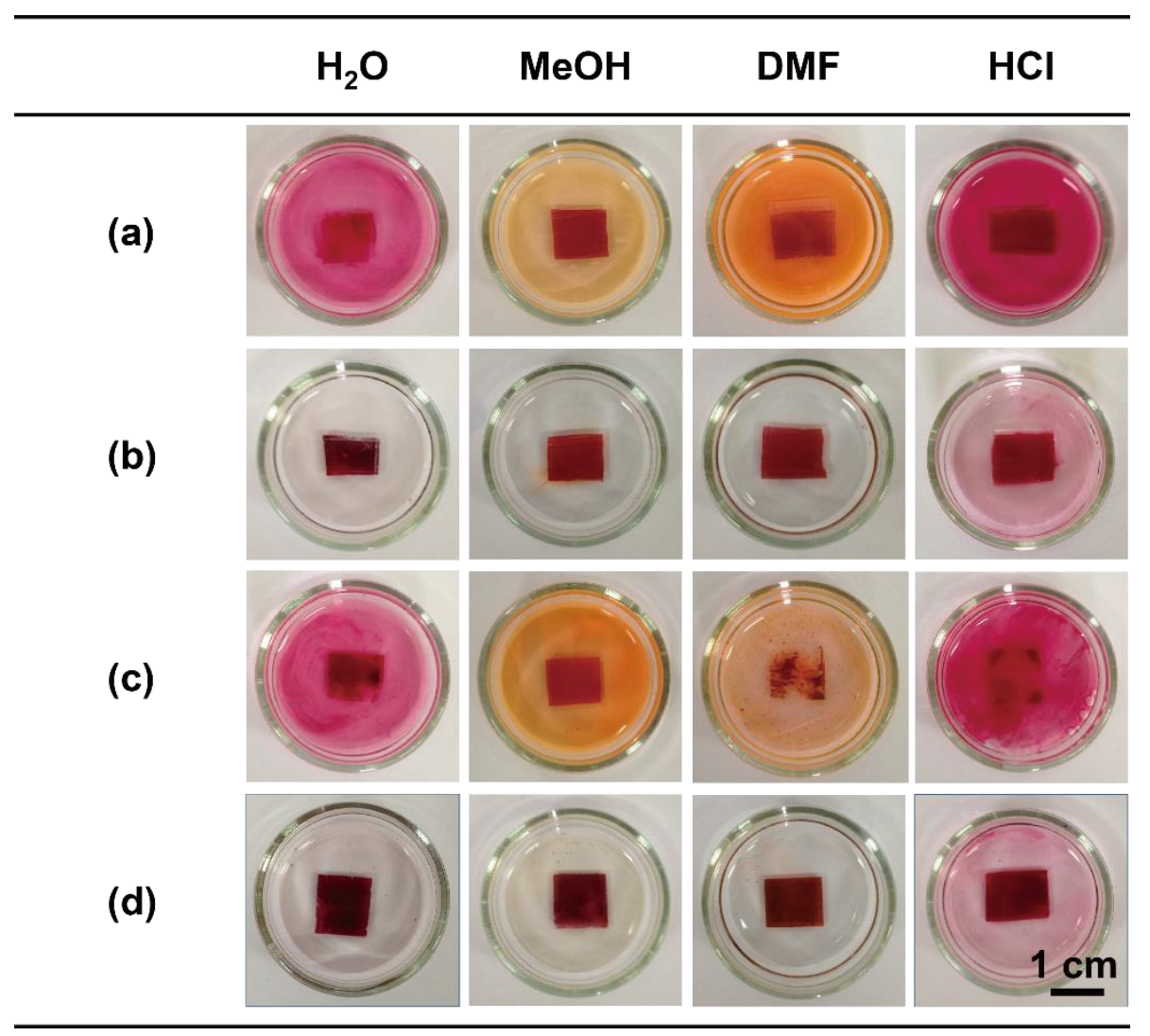

Figure S4. Photographic images after the chemical stability test of photo-polymerized DAPDI TFP on the (a) bare glass, (b) MAPT-modified glass, (c) bare PET and (d) MAPTmodified PET substrates: deionized water $\left(1^{\text {st }}\right.$ column $)$, methanol $\left(2^{\text {nd }}\right.$ column $)$, DMF $\left(3^{\text {rd }}\right.$ column) and $0.1 \mathrm{~N} \mathrm{HCl}\left(4^{\text {th }}\right.$ column$)$. Images are obtained after the 5 min dipping process in the solvents. 


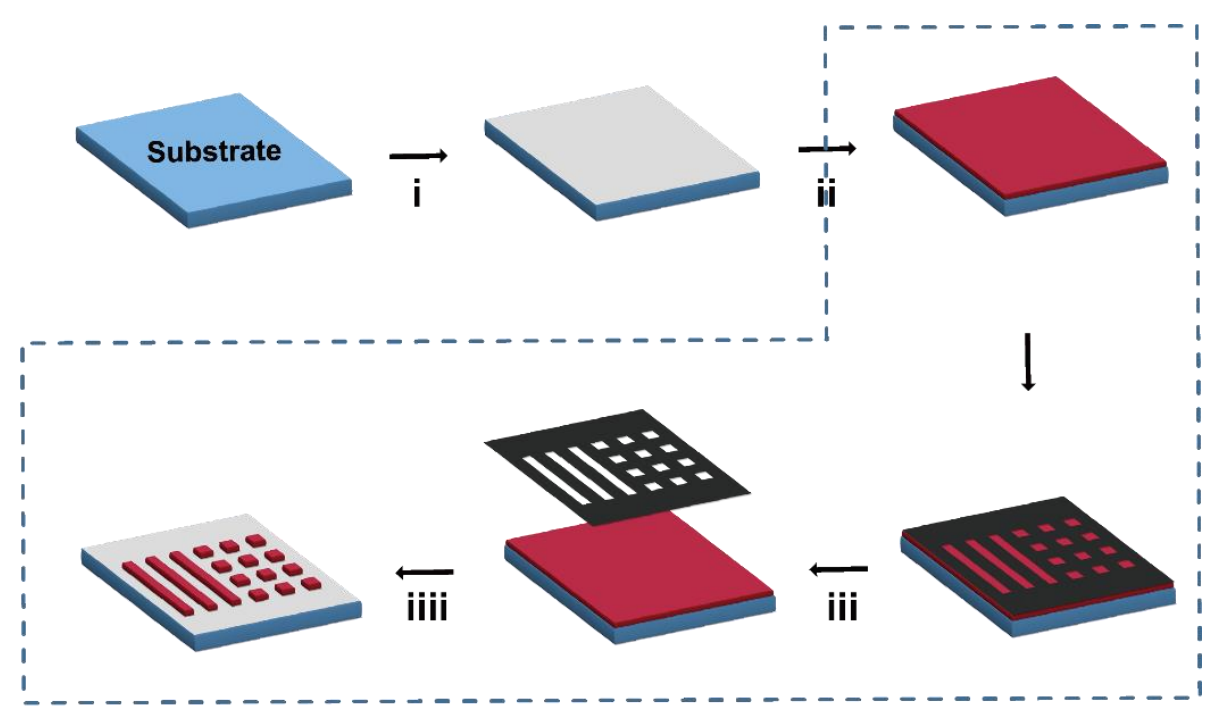

MAPT : Methacryloxypropyltrimethoxysilane $\stackrel{-0}{-0}-\mathrm{O}^{-}$

Figure S5. Fabrication of the patterned DAPDI TFP with lithographic methods: i) oxygen plasma, $5 \mathrm{~min}, 10 \mathrm{wt} \% \mathrm{MAPT} / \mathrm{EtOH}, 1 \mathrm{~h}$; ii) coating and drying, $2 \mathrm{~h}$; iii) UV irradiation, 30 $\min , 20 \mathrm{~mW} / \mathrm{cm}^{2}$; iiii) developed in methanol, $60 \mathrm{sec}$. Dashed line represented process for bare substrates. 

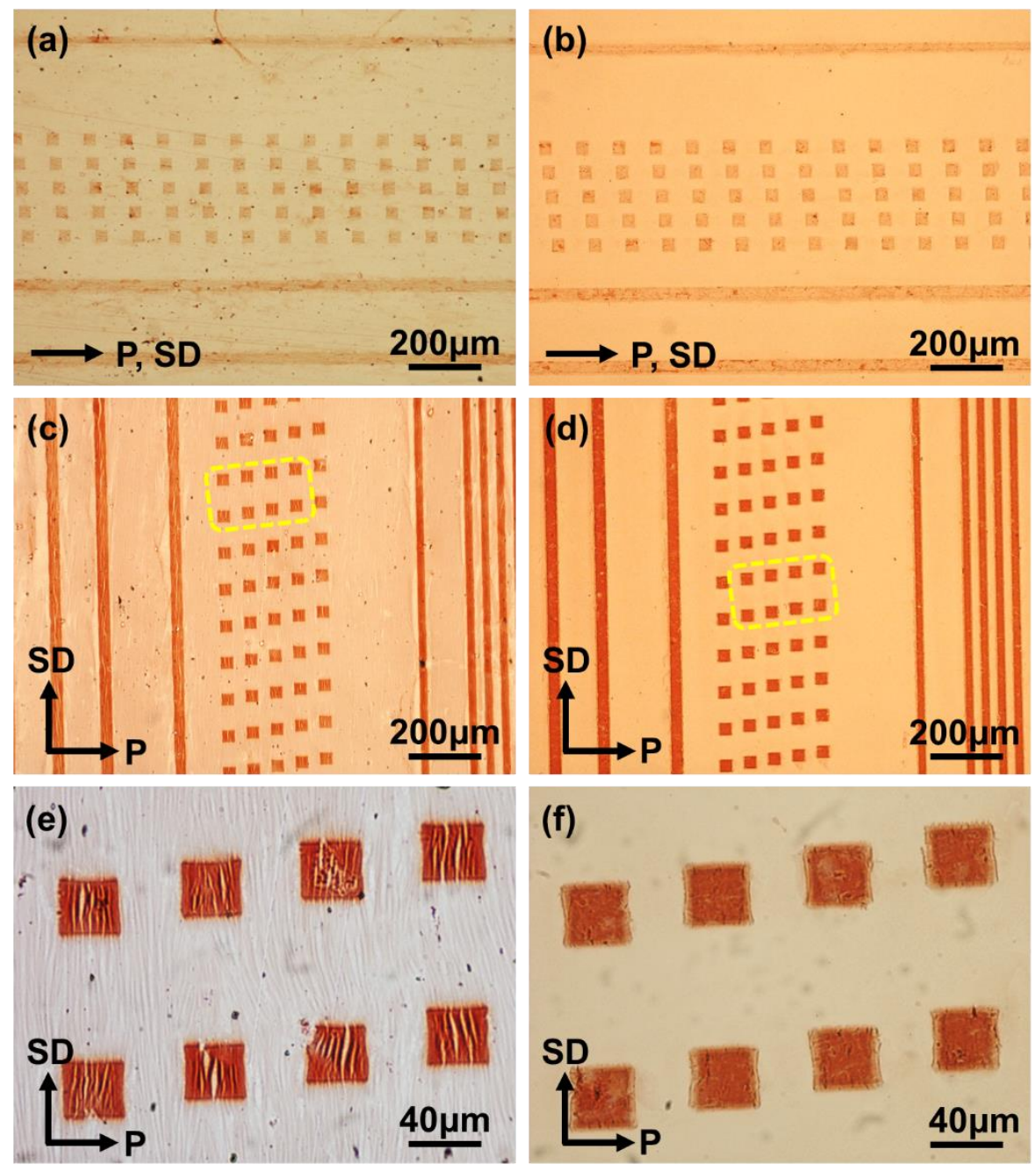

Figure S6. OM images of the positively patterned DAPDI TFP on the bare glass substrates when the polarizer axis is (a) parallel and (c) perpendicular to the SD and (e) its magnified image of dashed area of $6 c$. The corresponding OM images $(b, d, f)$ of patterned DAPDI TFP on the MAPT-modified glass substrate. 


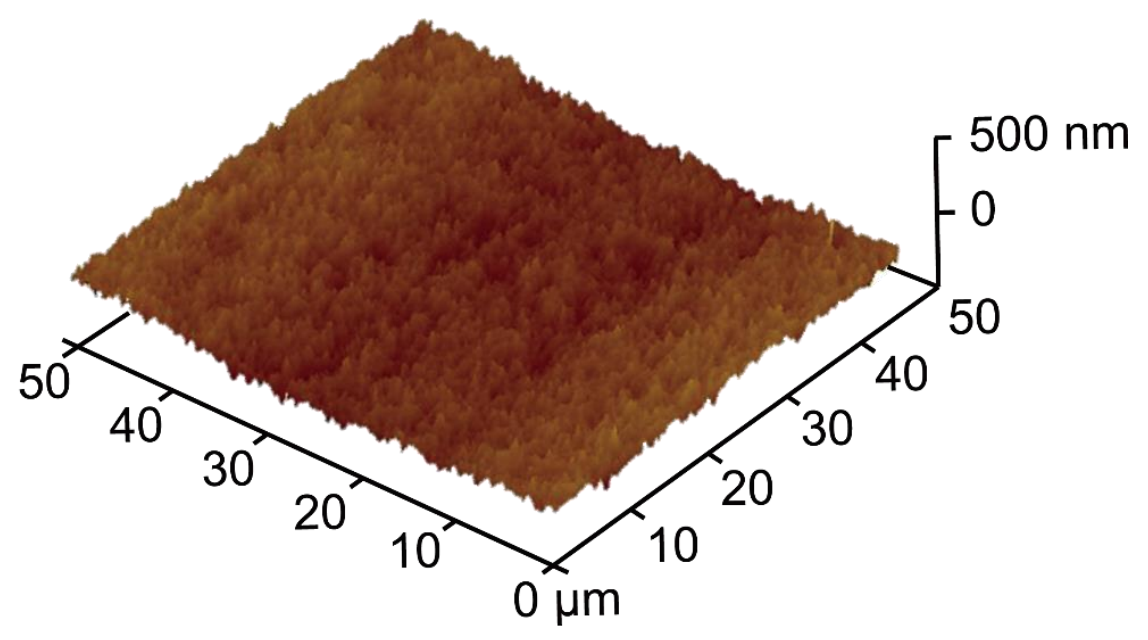

Figure S7. 3D topographic AFM image of the patterned DAPDI TFP on the MAPT-modified glass substrate. 